\section{Tamizaje de episodio depresivo en adolescentes. Validación del instrumento PHQ-9}

\author{
FRANCESCA BORGHERO ${ }^{1,2}$, VANIA MARTÍNEZ ${ }^{3,4}$, \\ PEDRO ZITKO ${ }^{5,6}$, PAUL A. VÖHRINGER ${ }^{4,7,8}$, \\ GABRIEL CAVADA ${ }^{1,9, a}$, GRACIELA ROJAS ${ }^{4,7}$
}

\section{Screening depressive episodes in adolescents. Validation of the Patient Health Questionnaire-9 (PHQ-9)}

Background: There is a paucity of validated instruments for screening depression in adolescent populations in Chile. Aim: To determine the diagnostic accuracy of the adolescent version of Patient Health Questionnaire-9 (PHQ-9). Material and Methods: The PHQ-9 was transculturally adapted and administered to adolescents aged 15 to 19 years residing in Santiago de Chile, who were then evaluated with a semi structured interview (Kiddie-Schedule for Affective Disorders and Schizophrenia-Present and Lifetime Version or K-SADS-PL) and the Beck Depression Inventory. Internal validity, concurrent validity, and discriminatory power of the PHQ-9 were analyzed. Results: We evaluated 245 adolescents aged $16.2 \pm 1$ years (71\% females). Two hundred and ten presented with a depressive episode and 35 were healthy. The sensitivity and specificity of the scale were 86.2 and $82.9 \%$ for 11 points, with a positive likelihood ratio of 5.02. Conclusions: The PHQ-9 is sensitive and specific enough to be used as a screening tool in adolescents with suspected depression. At a 11-point cut-off score as proposed, the likelihood to find a positive result in a subject with depression is five times higher.

(Rev Med Chile 2018; 146: 479-486)

Key words: Adolescent; Depression; Mass Screening; Patient Health Questionnaire; Primary Health Care.
${ }^{1}$ Programa de Magíster de Epidemiología Clínica, Universidad de Los Andes, Santiago, Chile.

${ }^{2}$ Servicio de Psiquiatría Infantil, Hospital Barros Luco Trudeau, Santiago, Chile.

${ }^{3}$ Centro de Medicina Reproductiva y Desarrollo Integral del Adolescente (CEMERA), Facultad de Medicina, Universidad de Chile, Santiago, Chile.

${ }^{4}$ Instituto Milenio para la Investigación en Depresión y Personalidad (MIDAP), Santiago, Chile.

${ }^{5}$ Unidad de Estudios Asistenciales, Complejo

Asistencial Barros Luco, Santiago, Chile. ${ }^{6}$ Health Services and Population Research Department, IoPPN, King's College London, Londres, Reino Unido.

${ }^{7}$ Departamento de Psiquiatría y Salud Mental, Hospital Clínico Universidad de Chile. Santiago, Chile.

${ }^{8}$ Mood Disorders Program, Tufts Medical Center, Tufts University, Boston, Estados Unidos.

9Departamento de Epidemiología, Universidad de los Andes, Santiago, Chile.

aphD en Bioestadística.

Fuente de apoyo financiera: Este estudio recibió el apoyo del FONDECYT de Iniciación No 11121637 y del Fondo de Innovación para la Competitividad (FIC) del Ministerio de Economía, Fomento y Turismo, a través de la Iniciativa Científica Milenio, Proyecto IS130005.

Recibido el 8 de septiembre de 2017 , aceptado el 5 de marzo de 2018.

Correspondencia a: Vania Martínez Nahuel

Centro de Medicina Reproductiva y Desarrollo Integral del Adolescente (CEMERA), Facultad de Medicina, Universidad de Chile. Profesor Alberto Zañartu 1030, Independencia, Santiago, Chile. vmartinezn@med.uchile.cl
$\mathrm{E}$ 1 episodio depresivo (ED) es una condición que se caracteriza por presencia de tristeza, pérdida de interés y de la capacidad de disfrutar, y aumento de la fatigabilidad entre los sujetos que la presentan ${ }^{1}$. Es una enfermedad que afecta a nuestra sociedad, provocando elevados costos individuales, sociales, familiares y económicos, además de influir de manera relevante en las cifras de muertes prematuras al asociarse con la conducta suicida ${ }^{2}$.
El ED puede presentarse en distintos grupos etarios. En niños y adolescentes se manifestará según el período evolutivo en el que los individuos se encuentren, observándose un incremento notorio en las cifras desde el inicio de la pubertad. Esto se asocia probablemente a cambios en su desarrollo cognitivo, en las estructuras cerebrales y hormonales, entre otros ${ }^{3}$.

El ED a edades tempranas y sin adecuado tratamiento tiende a ser debilitante y crónico, posibili- 
tando su persistencia y recurrencia en la adultez ${ }^{2}$. En la adolescencia, los cuadros de ED pueden asociarse a alteraciones en el funcionamiento y en el desarrollo psicosocial normal, manifestándose en dificultades escolares, problemas de relación con los padres y pares ${ }^{2}$. El ED constituye un riesgo de presentar trastornos por consumo de sustancias, conductas sexuales de riesgo, intentos suicidas y suicidios consumados ${ }^{2,4,5}$, y se acompaña de diversos grados de discapacidad y comorbilidad ${ }^{2,3}$.

En Chile, se estima que la prevalencia a nivel nacional para el ED en la población adolescente es de $8,3 \%{ }^{6}$. Al respecto, existe preocupación en relación a cifras que revelan que tan solo la mitad de ellos son pesquisados en esta etapa ${ }^{7} \mathrm{y}$, de este grupo, solo entre $20 \%$ y $50 \%$ accede finalmente a tratamiento ${ }^{6-9}$.

Por otra parte, el suicidio constituye la tercera causa de muerte en el tramo de edad comprendido entre los 10 y 24 años $^{8}$. Pese a la multiplicidad de factores asociados a la conducta suicida, la evidencia científica identifica al ED como uno de los más importantes ${ }^{10}$.

Debido a la relevancia del problema, el Ministerio de Salud de Chile ha propuesto, como objetivo sanitario para la década 2011-2020, reducir en $15 \%$ la tasa de mortalidad proyectada por suicidio en adolescentes (Objetivo Estratégico 4,3) ${ }^{10}$.

La detección e intervención precoz del ED durante la adolescencia impacta favorablemente en la calidad de la salud mental en la adultez ${ }^{5}$. De acuerdo con esto, los instrumentos de tamizaje para ED en este período son de suma relevancia. Además, si estos instrumentos son de fácil acceso, de aplicación breve y confiables, resultan ser herramientas útiles que aportan en la labor de detección temprana y tratamiento oportuno ${ }^{11}$.

Un instrumento destacado como con buenos desempeños en cuanto a sensibilidad y especificidad para detección del ED en adolescentes, e incluido en las recomendaciones de US Preventive Services Task Force, es el Patient Health Questionnaire-9 (PHQ-9) en su versión para adolescentes ${ }^{12-15}$. Este cuestionario forma parte del Patient Health Questionnaire-A (PHQ-A) ${ }^{5}$, que es la versión modificada para adolescentes de una herramienta de tamizaje empleada para los trastornos de salud mental y que es utilizada habitualmente en la atención primaria de salud (APS). Es una versión autoadministrada del Primary Care Evaluation of Mental Health Disorders $\left(\right.$ PRIME-MD) ${ }^{16}$ e incluye módulos dirigidos a evaluar trastornos del ánimo, trastornos de ansiedad, trastornos de la conducta alimentaria, trastornos somatomorfos y abuso de alcohol y otras sustancias ${ }^{17}$. El PHQ-9, al ser autoaplicado, puede utilizarse en APS, optimizando los tiempos de evaluación de pacientes consultantes en este nivel de atención ${ }^{18,19}$. Este cuestionario ha sido traducido y validado en diferentes idiomas con adecuada validez diagnóstica ${ }^{20,21}$. En Chile fue validado para población adulta por Baader et $\mathrm{a}^{18}$ y en Estados Unidos de Norteamérica ${ }^{11}$ cuenta con una versión para población adolescente. El presente trabajo pretende determinar la validez de la versión adaptada para adolescentes y así contribuir a mejorar la pesquisa de ED en este grupo etario.

\section{Material y Método}

\section{Adaptación transcultural}

El PHQ-9 se adaptó transculturalmente para Chile mediante un proceso de dos traducciones directas desde la versión original en inglés. Se realizó una conciliación de ambas versiones traducidas, una retrotraducción al inglés y una comparación con la versión original. Posteriormente, se realizó entrevistas cognitivas a una muestra de adolescentes sanos con el fin de evaluar la comprensión de las preguntas.

\section{Diseño y participantes}

Este trabajo corresponde a un estudio diagnóstico de corte transversal.

La muestra se obtuvo mediante un muestreo no probabilístico con reclutamiento consecutivo de adolescentes de 15 a 19 años usuarios de cuatro centros de salud familiar de la comuna de Puente Alto, Santiago de Chile, además de alumnos regulares de cuatro colegios particulares subvencionados de la misma comuna y de la comuna de Pedro Aguirre Cerda, también de la ciudad de Santiago. La muestra fue recolectada entre octubre de 2013 y mayo de 2016 . Se excluyeron adolescentes que reportaran, al momento de la evaluación, uso de antidepresivos o estar en psicoterapia por ED; adolescentes mujeres que reportaran embarazo o hijo menor de un año; y adolescentes que clínicamente evidenciaran discapacidad intelectual y comorbilidad psiquiátrica (trastorno bipolar, psicosis o trastorno por abuso 
de sustancias), lo que fue evaluado usando el Schedule for Affective Disorders and Schizophrenia for School-Age Children-Present and Lifetime version (Kiddie-SADS-PL) ${ }^{22}$.

\section{Instrumentos y recolección de la información}

Se aplicó el cuestionario PHQ-9 en su versión para adolescentes (Figura 1), el cual consta de nueve preguntas destinadas a evaluar sintomatología depresiva según criterios DSM, en una escala ordinal según frecuencia de síntomas (nunca, algunos días, más de la mitad de los días, casi todos los días). El rango de puntuación abarca desde el 0 a los 27 puntos, donde mayores

\section{PHQ-9 versión para adolescentes}

Nombre:

Fecha:

Instrucciones: ¿Con qué frecuencia te han incomodado alguno de los siguientes síntomas durante las últimas dos semanas?

(Para cada síntoma marca con una "x" la respuesta que mejor describe cómo te has sentido)

\begin{tabular}{|c|c|c|c|c|}
\hline & Nunca (0) & $\begin{array}{c}\text { Algunos días } \\
(1)\end{array}$ & $\begin{array}{l}\text { Más de la } \\
\text { mitad de los } \\
\text { días (2) }\end{array}$ & $\begin{array}{l}\text { Casi todos } \\
\text { los días (3) }\end{array}$ \\
\hline \multicolumn{5}{|l|}{$\begin{array}{l}\text { 1. ¿Te has sentido bajoneado/a, deprimido/a, irritable o } \\
\text { desesperanzado/a? }\end{array}$} \\
\hline \multicolumn{5}{|l|}{ 2. ¿Has sentido poco interés o placer al hacer las cosas? } \\
\hline \multicolumn{5}{|l|}{$\begin{array}{l}\text { 3. ¿ंHas sentido problemas para quedarte dormido/a, permanecer } \\
\text { dormido/a, o has estado durmiendo demasiado? }\end{array}$} \\
\hline \multicolumn{5}{|l|}{ 4. ¿Te has sentido cansado/a o con poca energía? } \\
\hline \multicolumn{5}{|l|}{$\begin{array}{l}\text { 5. ¿Has tenido poco apetito, has bajado de peso, o has comido } \\
\text { excesivamente? }\end{array}$} \\
\hline \multicolumn{5}{|l|}{$\begin{array}{l}\text { 6. ¿Te has sentido mal respecto a ti mismo/a o has sentido que tu eres } \\
\text { un/a fracasado/a, o que has decepcionado a tu familia o a ti mismo/a? }\end{array}$} \\
\hline \multicolumn{5}{|l|}{$\begin{array}{l}\text { 7. ¿Has tenido problemas para concentrarte en actividades como } \\
\text { trabajos escolares, leer, o ver televisión? }\end{array}$} \\
\hline \multicolumn{5}{|l|}{$\begin{array}{l}\text { 8. ¿Te has movido o hablado tan lento que las otras personas podrían } \\
\text { haberlo notado? } \mathrm{O} \text { al contrario ¿has estado tan inquieto/a que estabas } \\
\text { moviéndote de un lado para otro mucho más de lo usual? }\end{array}$} \\
\hline $\begin{array}{l}\text { 9. ¿Has pensado que sería mejor estar muerto/a o has pensado hacerte } \\
\text { daño de alguna manera? }\end{array}$ & & & & \\
\hline
\end{tabular}

a. En el último año, ¿te has sentido deprimido o triste la mayoría de los Sí

días, aunque te sientas a veces bien?

No

Ninguna dificultad Algo de dificultad

b. Si estás experimentando alguno de los problemas de este $\begin{array}{ll}\text { cuestionario, ¿cuánto hacen esos problemas que se te dificulte hacer tu Bastante dificultad } \\ \text { trabajo, tus labores en la casa, o llevarte bien con los demás? } & \text { Extrema Dificultad }\end{array}$

c. Durante étimo mes ihaspensado en aun momentoserlamente si

c. Durante el último mes ¿has pensado en algún momento seriamente Sí en terminar con tu vida?

d. Alguna vez en tu vida, ¿has tratado de matarte o has hecho un intento de suicidio?

\begin{tabular}{ll} 
Sí & \\
No & \\
\hline Sí & \\
No & \\
\hline
\end{tabular}

Si has tenido pensamientos de que sería mejor estar muerto/a o has pensado en hacerte daño de alguna manera, por favor convérsalo con el/la profesional que está a cargo de tu caso.

Figura 1. Patient Health Questionnaire 9 versión para Adolescentes. Modificado con la autorización PHQ (Spitzer, William \& Kroenke, 1999) por J. Johnson (Johnson, 2002). Adaptación y validación al castellano para Chile por Francesca Borghero, Vania Martínez, Pedro Zitko, Paul Vöhringer, Gabriel Cavada y Graciela Rojas. 
valores indican mayor probabilidad de ED. El cuestionario, adicionalmente, presenta cuatro preguntas complementarias destinadas a evaluar (A) temporalidad del ánimo depresivo, (B) grado de disfuncionalidad que genera la sintomatología, (C) ideas e (D) intentos suicidas ${ }^{5,11,23}$.

Asimismo, de la muestra se recolectaron datos sociodemográficos, además de aplicar el Inventario de Depresión de Beck (BDI) ${ }^{24}$, instrumento que explora sintomatología depresiva presente en la última semana en una escala ordinal según gravedad de los síntomas (21 ítems, puntaje entre 0 y 63). Además, cada participante fue evaluado por un psiquiatra infantil y del adolescente, o psicólogo entrenado, quienes aplicaron la entrevista Kiddie-SADS-PL para la confirmación diagnóstica o descarte del ED. Esta corresponde a una entrevista diagnóstica semiestructurada basada en los criterios DSM-III-R y DSM-IV para evaluar episodios actuales y pasados de 46 diagnósticos psiquiátricos en niños y adolescentes ${ }^{22}$. Para este estudio se utilizaron los módulos de tamizaje y suplementos de dicha entrevista para confirmar ED y excluir casos de trastorno bipolar, psicosis y abuso de sustancias.

\section{Análisis estadístico}

El cálculo del tamaño de muestra ad hoc para conseguir una sensibilidad de $90 \%$, especificidad de $80 \%{ }^{5,25}$, considerando una prevalencia de $8,3 \%$ para ED en adolescentes entre 12 y 18 años $^{6}$, estableciendo un nivel de significación de $1 \%$ y una potencia de 0,9 , correspondió a un total de 200 adolescentes, con un mínimo de 25 adolescentes sanos como controles.

Las variables categóricas se describieron como frecuencias y porcentajes. Las variables numéricas se describieron por el mínimo, máximo, promedio y desviación estándar.

Se consideraron que valores del alfa de Cronbach superiores a 0,70 eran suficientes para garantizar la consistencia interna del instrumento.

La asociación entre los puntajes de PHQ-9 y BDI fue evaluada a través de coeficiente de correlación de Pearson. La capacidad de discriminación del PHQ-9 para depresión se evaluó mediante el área bajo la curva ROC (Receiver Operating Characteristic), con su respectivo intervalo de confian$\mathrm{za}$, tanto en forma cruda como ajustada por sexo. La comparación entre áreas bajo la curva ROC se hizo mediante el test DeLong, Clark-Pearson ${ }^{26}$. Los intervalos de confianza fueron de nivel 95\%, se utilizó una significación de $5 \%$ y los datos fueron procesados en STATA versión 14.0.

\section{Consideraciones éticas}

El presente proyecto fue aprobado para su desarrollo por el Comité de Ética de la Investigación en Seres Humanos de la Facultad de Medicina de la Universidad de Chile (Proyecto No 145-2013).

Los instrumentos mencionados fueron aplicados luego de firmado el consentimiento informado del adolescente (para el caso de los menores de edad se requirió consentimiento informado de los padres o tutor legal y asentimiento del adolescente para participar en el estudio). La participación en el estudio fue voluntaria, pudiendo desvincularse de la evaluación si así lo estimaban pertinente, sin que ello afectase su atención habitual. Los casos con alto riesgo suicida o con diagnóstico de ED fueron derivados para recibir atención. El uso de los datos fue manejado de manera confidencial.

\section{Resultados}

\section{Adaptación transcultural}

Se entrevistó a 14 adolescentes sanos entre $15 \mathrm{y}$ 18 años. El 43\% $(n=6)$ eran mujeres. Se evidenció adecuada compresión de las preguntas de las versión traducida y aplicada del PHQ-9.

\section{Resultados de la muestra}

Se contactó a 250 adolescentes y, de ellos, aceptaron ser entrevistados 245 , entre 15 y 19 años, con un promedio de edad de 16,2 (DE 1,1) años. El $71 \%(\mathrm{n}=174)$ fueron mujeres. El rango de escolarización efectiva fue entre quinto básico y cuarto medio con un promedio de 9,8 (DE 1,5) años completados. El 14,3\% ( $\mathrm{n}=35)$ de la muestra correspondió a adolescentes sanos. En la Tabla 1 se muestran los datos sociodemográficos de los participantes según la condición de presentar ED.

\section{Aplicación PHQ-9}

Los puntajes obtenidos entre la pregunta 1 y la 9 según condición de ED del PHQ-9 se muestran en la Tabla 2.

Respecto a la pregunta de temporalidad de la sintomatología, destaca que 95\% (n = 199) de los 
Tabla 1. Datos sociodemográficos de los participantes $(\mathbf{n}$ total $=245$ )

\begin{tabular}{|c|c|c|c|c|c|}
\hline \multirow[t]{2}{*}{ Variable } & \multicolumn{2}{|c|}{ Sanos $(n=35)$} & \multicolumn{2}{|c|}{ Episodio depresivo $(n=210)$} & \multirow[t]{2}{*}{ Valor $p$} \\
\hline & $\mathbf{n}$ & $\%$ & $\mathbf{n}$ & $\%$ & \\
\hline \multirow[t]{2}{*}{ Sexo femenino } & 15 & 43 & 159 & 76 & 0,000 \\
\hline & Media & DE & Media & DE & Valor $p$ \\
\hline Edad & 16,1 & 1,19 & 16,3 & 1,08 & 0,159 \\
\hline Años escolaridad & 9,7 & 1,9 & 9,8 & 1,42 & 0,357 \\
\hline
\end{tabular}

Tabla 2. Puntaje por pregunta del instrumento según condición de episodio depresivo

\begin{tabular}{|c|c|c|c|c|}
\hline \multirow[t]{2}{*}{$\begin{array}{c}N^{\circ} \text { de } \\
\text { pregunta }\end{array}$} & \multicolumn{4}{|c|}{$\begin{array}{l}\text { Puntaje según condición } \\
\text { Sano } \\
\begin{array}{l}\text { Ep. depresivo } \\
(n=35)\end{array} \\
(n=210)\end{array}$} \\
\hline & $\begin{array}{c}\text { Media } \\
\text { (DE) }\end{array}$ & Min-Max & $\begin{array}{c}\text { Media } \\
\text { (DE) }\end{array}$ & Min-Max \\
\hline P1 & $\begin{array}{c}1,06 \\
(0,48)\end{array}$ & $0-2$ & $\begin{array}{c}2,28 \\
(0,83)\end{array}$ & $0-3$ \\
\hline P2 & $\begin{array}{l}0,97 \\
(71)\end{array}$ & $0-3$ & $\begin{array}{c}2,02 \\
(0,84)\end{array}$ & $0-3$ \\
\hline P3 & $\begin{array}{l}1,17 \\
(1,1)\end{array}$ & $0-3$ & $\begin{array}{c}2,12 \\
(1,04)\end{array}$ & $0-3$ \\
\hline P4 & $\begin{array}{c}1,23 \\
(0,94)\end{array}$ & $0-3$ & $\begin{array}{c}2,22 \\
(0,82)\end{array}$ & $0-3$ \\
\hline P5 & $\begin{array}{c}0,94 \\
(0,87)\end{array}$ & $0-3$ & $\begin{array}{c}1,78 \\
(1,03)\end{array}$ & $0-3$ \\
\hline P6 & $\begin{array}{c}0,69 \\
(0,72)\end{array}$ & $0-2$ & $\begin{array}{c}1,8 \\
(1,0)\end{array}$ & $0-3$ \\
\hline P7 & $\begin{array}{c}1,23 \\
(0,94)\end{array}$ & $0-3$ & $\begin{array}{c}2,01 \\
(1,05)\end{array}$ & $0-3$ \\
\hline P8 & $\begin{array}{c}0,37 \\
(0,69)\end{array}$ & $0-3$ & $\begin{array}{l}1,40 \\
(1,0)\end{array}$ & $0-1$ \\
\hline P9 & $\begin{array}{c}0,11 \\
(0,32)\end{array}$ & $0-1$ & $\begin{array}{c}1,12 \\
(0,88)\end{array}$ & $0-3$ \\
\hline Total & $\begin{array}{l}7,77 \\
(3,5)\end{array}$ & $0-17$ & $\begin{array}{l}16,76 \\
(5,14)\end{array}$ & $5-27$ \\
\hline
\end{tabular}

encuestados con ED se sintió triste/deprimido la mayoría de los días en el último año. El 51\% $(\mathrm{n}=18)$ de los sanos no experimentó sentirse triste/deprimido la mayoría de los días en el último año.

En relación a las dificultades que generó la sintomatología, $40 \%(\mathrm{n}=14)$ de los encuestados sanos no reportaron presentar dificultades en la realización de sus actividades diarias, mientras que $57 \%(\mathrm{n}=20)$ de ellos presentó algo de dificultad. Ninguno de ellos reportó extrema dificultad. El $100 \%(\mathrm{n}=12)$ de los que reportaron dificultad extrema presentaban ED.

El 91\% $(n=32)$ de los sanos y 60\% $(n=126)$ de los encuestados con ED negaron haber pensado seriamente en el último mes en terminar con su vida. Respecto al total de los encuestados que sí pensaron seriamente en el último mes en terminar con su vida, $97 \%(\mathrm{n}=83)$ correspondió a sujetos con ED.

Al explorar los intentos suicidas, $36 \%(n=89)$ de los encuestados presentó algún intento suicida en su vida, de ellos $94 \%(n=84)$ correspondieron a adolescentes con ED.

\section{Desempeño diagnóstico del PHQ-9}

En el análisis de consistencia interna el PHQ-9 obtuvo un valor del alfa de Cronbach de 0,78 (IC 95\%: 0,71-0,84), lo cual garantiza la fiabilidad del instrumento.

En relación a la validez concurrente, esta se evaluó mediante la correlación del PHQ-9 con el BDI. Se encontró un nivel de correlación de 0,72 (IC 95\%: 0,64-0,78). En la Figura 2 se expone el diagrama de dispersión entre los resultados del PHQ-9 y el BDI, lo cual evidenció una alta correlación positiva entre ambos instrumentos.

Respecto a la capacidad diagnóstica, se observó que por cada punto adicional de PHQ-9 aumentaba en 1,5 veces la probabilidad de presentar un ED según el gold standard empleado (OR: 1,50 IC 95\%: 1,32-1,72). Con 11 puntos se alcanzó una sensibilidad de $86,2 \%$ y una especificidad de $82,9 \%$, con una razón de probabilidad positiva de 5,02, siendo 5 veces más probable encontrar un resultado positivo en un sujeto con depresión respecto a uno sin el cuadro. 

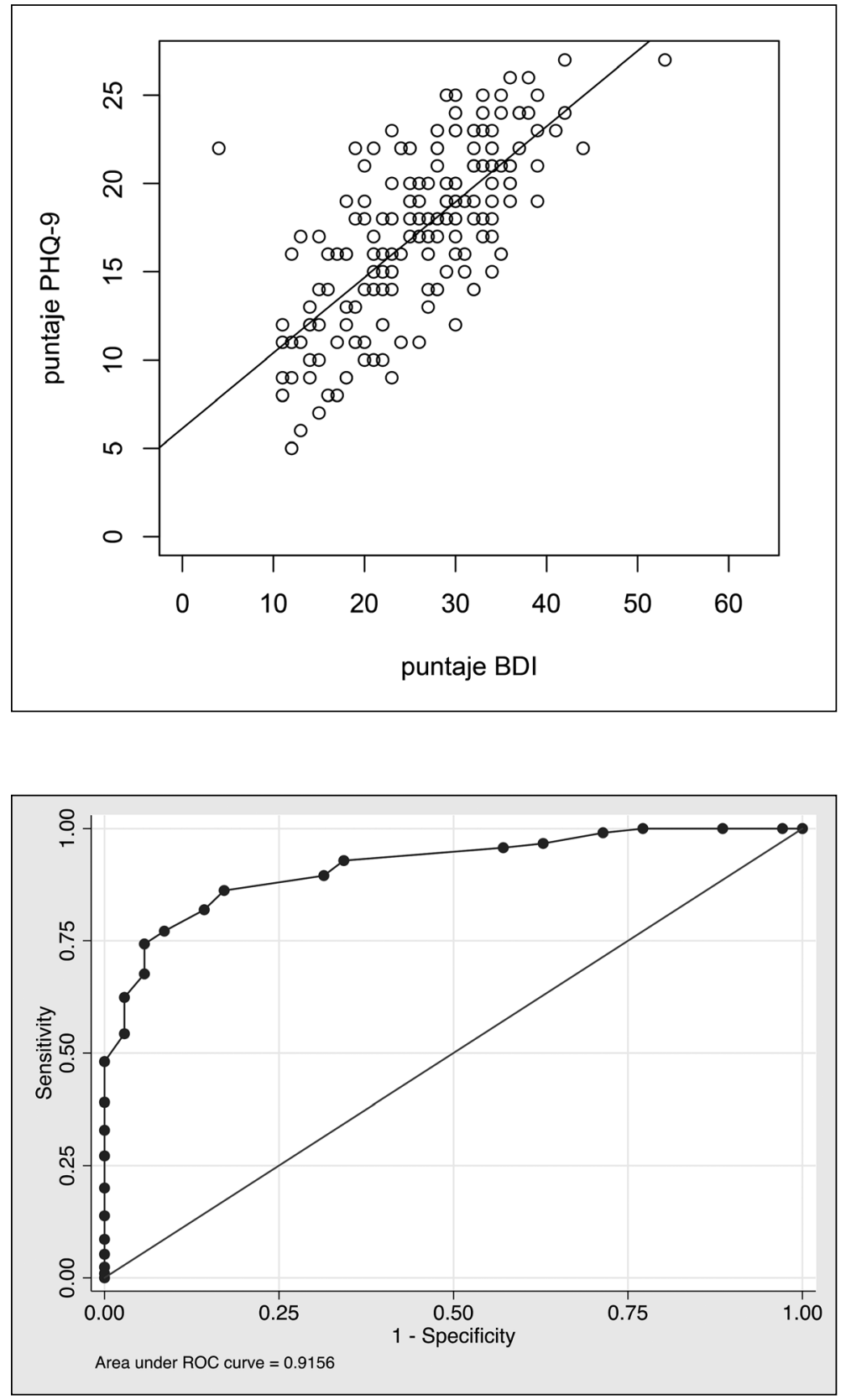

Figura 2. Dispersión entre $\mathrm{BDI}$ y $\mathrm{PHQ}-$ 9. Nivel de correlación de 0,72 (IC 95\%: $0,64-0,78)$.

Figura 3. Curva ROC (Receiver Operating Characteristic) para PHQ-9.

La capacidad de discriminación fue de 92\% (área bajo la curva ROC con IC 95\%: 87\%-96\%) (Figura 3). La capacidad de discriminación en hombres fue $86 \%$ (CI 95\%: 77\%-94\%), mientras que en mujeres fue de 92\% (CI 95\%: 86\%-99\%), sin evidenciarse diferencias estadísticamente significativas $(\mathrm{p}=0,140)$.

\section{Discusión}

Los resultados de este estudio señalan que el PHQ-9 presenta adecuadas propiedades para la pesquisa de ED en adolescentes chilenos. Adicionalmente, este ha sido recomendado por el US Preventive Services Task Force para el tamizaje de 
ED en adolescentes ${ }^{14,15}$. Tanto la versión validada en adultos chilenos ${ }^{18}$, como la versión original en inglés, muestran sensibilidades y especificidades adecuadas para ser implementados con este $\mathrm{fin}^{11}$.

Entre las fortalezas del estudio se encuentra una validación que incluye aspectos transculturales y evaluaciones a cada adolescente realizado por personal entrenado en Kiddie-SADS-PL. En relación a la validez concurrente, el PHQ-9 demuestra un alto nivel de correlación con el BDI, lo cual lo hace ser un instrumento óptimo para ser aplicado en APS en adolescentes, pues se trata de un instrumento breve, autoaplicado y de uso gratuito.

El puntaje de corte que se propone para ED es de 11 o más. Con 11 puntos se obtiene una razón de probabilidad positiva de 5,02, lo cual sitúa al PHQ-9 como una herramienta útil para tomar decisiones clínicas. Estos son valores inherentes al instrumento e independientes de la prevalencia de ED en adolescentes.

La capacidad de discriminación fue de $92 \%$ en el análisis del área bajo la curva de ROC (IC 95\%: 87\%-96\%), sin evidenciarse diferencias estadísticamente significativas al considerar la variable sexo en este análisis $(\mathrm{p}=0,140)$. La capacidad de discriminación en hombres fue 86\% (CI 95\%: $77 \%-94 \%$ ) mientras que en mujeres fue de $92 \%$ (CI 95\%: 86\%-99\%). En este aspecto es importante señalar posibles limitaciones del estudio, pues si bien se cumplió con el tamaño muestral propuesto para sanos y enfermos, la proporción de evaluados entre hombres y mujeres fue dispar al considerar que $71 \%$ de la muestra correspondió a adolescentes mujeres.

Desde una mirada de salud pública, el PHQ-9 es un instrumento que puede contribuir a mejorar la atención de salud mental en adolescentes, optimizando la detección de ED en este grupo etario. Esto es de especial relevancia si se considera la escasez de especialistas en Psiquiatría infantil y del adolescente en Chile, quienes, además, se encuentran distribuidos de manera desigual a lo largo del país ${ }^{27}$, situación que dificulta la detección y el tratamiento del ED en adolescentes ${ }^{6-9}$.

De acuerdo con lo anterior, sería relevante continuar con una validación del instrumento abarcando períodos más tempranos de la adolescencia y ampliar los resultados para evaluar el desempeño del instrumento como herramienta de seguimiento del ED. No obstante, existe evidencia del uso adecuado del PHQ-9 desde los 12 años $^{5,11,20,23}$, así como también durante el tratamiento del $\mathrm{ED}^{17}$.

En síntesis, y de acuerdo con los resultados expuestos, el PHQ-9 constituye una herramienta útil, confiable y de fácil acceso para ser aplicado en adolescentes consultantes de la APS para la pesquisa oportuna del ED.

Agradecimientos: Este estudio recibió el apoyo del FONDECYT de Iniciación No 11121637 y del Fondo de Innovación para la Competitividad (FIC) del Ministerio de Economía, Fomento y Turismo, a través de la Iniciativa Científica Milenio, Proyecto IS130005. Los autores agradecen al Área de Salud de la Corporación Municipal de Puente Alto, a los directores de los centros de salud de atención primaria, a los directores de establecimientos educacionales y a los adolescentes que participaron en este estudio.

\section{Referencias}

1. Asociación Americana de Psiquiatría. Trastornos depresivos. En: Asociación Americana de Psiquiatría, Guía de Consulta de los criterios diagnósticos del DSM 5. Arlington, VA; 2013. p. 104-8.

2. Cook MN, Peterson J, Sheldon C. Adolescent depression: An update and guide to clinical decision making. Psychiatry Edgmont 2009; 6 (9): 17-31.

3. Irribarne M. Desarrollo psicológico del adolescente. En: Almonte C, Montt M. Psicopatología infantil y de la adolescencia. Segunda Edición. Santiago, Chile: Editorial Mediterráneo Ltda.; 2012. p. 37-47.

4. Castro A, Larraín A, Fritsch R, Rojas G. Telepsiquiatría: una revisión sistemática cualitativa. Rev Med Chile 2012; 140 (6): 789-96.

5. Johnson J, Harris E, Spitzer R, Williams J. The patient health questionnaire for adolescents: validation of an instrument for the assessment of mental disorders among adolescent primary care patients. J Adolesc Health Off Publ Soc Adolesc Med 2002; 30 (3): 196-204.

6. Vicente B, Saldivia S, de la Barra F, Melipillán R, Valdivia M, Kohn R. Salud mental infanto-juvenil en Chile y brechas de atención sanitarias. Rev Med Chile 2012; 140 (4): 447-57.

7. Kessler RC, Avenevoli S, Ries Merikangas K. Mood disorders in children and adolescents: an epidemiologic perspective. Biol Psychiatry 2001; 49 (12): 1002-14.

8. Hakak R, Szeftel R. Clinical Use of Telemedicine in Child Psychiatry. Focus J Lifelong Learn Psychiatry 2008; 6 (3): 293-6. 
9. Nelson E, Barnard M, Cain S. Treating childhood depression over videoconferencing. Telemed J E Health 2003; 9 (1): 49-55.

10. Ministerio de Salud de Chile (MINSAL). 2011. Estrategia Nacional de Salud para el Cumplimiento de los Objetivos Sanitarios de la Década 2011 - 2020. Disponible en: http://www.minsal.gob.cl.

11. Richardson L, McCauley E, Grossman D, McCarty C, Richards J, Russo J, et al. Evaluation of the Patient Health Questionnaire-9 Item for detecting major depression among adolescents. Pediatrics 2010; 126 (6): 1117-23.

12. Maurer D. Screening for depression. Am Fam Physician 2012; 85 (2): 139-44.

13. Hamrin V, Magorno M. Assessment of adolescents for depression in the pediatric primary care setting. Pediatr Nurs 2010; 36 (2): 103-11.

14. US Preventive Services Task Force. Screening and Treatment for Major Depressive Disorder in Children and Adolescents: US Preventive Services Task Force Recommendation Statement. Pediatrics 2009; 123 (4): 1223-8.

15. Siu A, US Preventive Services Task Force. Screening for Depression in Children and Adolescents: US Preventive Services Task Force Recommendation Statement. Pediatrics 2016; 137 (3): e20154467.

16. Spitzer R, Williams J, Kroenke K, Linzer M, deGruy F, Hahn S, et al. Utility of a new procedure for diagnosing mental disorders in primary care. The PRIME-MD 1000 study. JAMA 1994; 272 (22): 1749-56.

17. PHQ Screeners. Instructions for Patient Health Questionnaire (PHQ) and GAD-7 Measures [Internet]. Disponible en: http://www.phqscreeners.com

18. Baader T, Molina J, Venezian S, Rojas C, Farías R, Fierro-Freixenet $\mathrm{C}$, et al. Validación y utilidad de la encuesta PHQ-9 (Patient Health Questionnaire) en el diagnóstico de depresión en pacientes usuarios de atención primaria en Chile. Rev Chil Neuro-Psiquiatr 2012;
50 (1): 10-22.

19. Hansson M, Chotai J, Nordstöm A, Bodlund O. Comparison of two self-rating scales to detect depression: HADS and PHQ-9. Br J Gen Pract J R Coll Gen Pract 2009; 59 (566): e283-8.

20. Allgaier A, Pietsch K, Frühe B, Sigl-Glöckner J, Schulte-Körne G. Screening for depression in adolescents: validity of the patient health questionnaire in pediatric care. Depress Anxiety 2012; 29 (10): 906-13.

21. Gilbody S, House A, Sheldon T. Screening and case finding instruments for depression. Cochrane Database Syst Rev Online 2005; (4): CD002792.

22. Kaufman J, Birmaher B, Brent D, Rao U, Flynn C, Moreci $\mathrm{P}$, et al. Schedule for Affective Disorders and Schizophrenia for School-Age Children-Present and Lifetime Version (K-SADS-PL): initial reliability and validity data. J Am Acad Child Adolesc Psychiatry 1997; 36 (7): 980-8.

23. Wittkampf KA, Naeije L, Schene AH, Huyser J, van Weert HC. Diagnostic accuracy of the mood module of the Patient Health Questionnaire: a systematic review. Gen Hosp Psychiatry 2007; 29 (5): 388-95.

24. Beck A, Steer R, Carbin M. Psychometric properties of the Beck Depression Inventory: Twenty-five years of evaluation. Clin Psychol Rev 1988; 8 (1): 77-100.

25. Williams S, O'Connor E, Eder M, Whitlock E. Screening for Child and Adolescent Depression in Primary Care Settings: A Systematic Evidence Review for the US Preventive Services Task Force. Pediatrics 2009; 123 (4): e716-35.

26. DeLong ER, DeLong DM, Clarke-Pearson DL. Comparing the areas under two or more correlated receiver operating characteristic curves: a nonparametric approach. Biometrics 1988; 44 (3): 837-45.

27. Ministerio de Salud de Chile. Estudio de Brechas de Oferta y Demanda de Médicos Especialistas en Chile. 2010. 